ARTÍCULO ESPECIAL / SPECIAL ARTICLE

\title{
Estudio de cohortes prospectivo en etapa temprana de la pandemia COVID-19, Partido de General Pueyrredón, Argentina (INECOVID): dinámica de infección y factores de riesgo
}

\section{Prospective cohort study in the early stage of the COVID-19 pandemic, General Pueyrredón, Argentina (INECOVID): infection dynamics and risk factors}

\author{
Jimena Marro' (D), Christian Ballejo' (D), María Fernanda Aguirre' (D), \\ María Eugenia de San Martín' (iD, Lucía López Miranda' (D), Verónica Poncet' (iD, Andrea Silva'
}

RESUMEN: Objetivo: Establecer la magnitud y los factores de riesgo de infección por SARS-CoV-2 en el Partido de General Pueyrredón, Buenos Aires, Argentina: estudio INECOVID. Métodos: Diseño de cohortes prospectivo con participantes de población general del partido. El período de seguimiento fue del 22 de junio al 18 de diciembre de 2020, con un intervalo mínimo de citación de 21 días. Los datos se obtuvieron mediante cuestionarios y muestras de suero o plasma. El evento primario fue el tiempo hasta la seroconversión (IgG) como evidencia de infección por SARS-CoV-2. Se estimó el riesgo acumulado de infección por el método de Kaplan Meier. Se construyeron modelos de Cox con variables tiempo-dependientes. Resultados: Fueron reclutados 345 participantes ( $n=222$ mujeres, 64,3\%; 123 hombres, 35,7\%), con una edad mediana de 45 años en mujeres (Rango intercuartílico: 19) y 49 en hombres (Rango intercuartílico: 26). El 12,8\% de los participantes (n=44) tuvieron evidencia de infección por SARS-CoV-2 [densidad de incidencia de 9,1 casos (mujeres: 11,1, hombres: $5,1)$ por 10.000 personas-días]. El 36,4\% de los casos $(n=16)$ fueron asintomáticos. Se mostraron asociados al riesgo de infección: ser contacto estrecho de un caso confirmado de COVID-19 (HR=5,56; IC95\% 2,85-10,83), ser trabajador de salud (HR=2,93; IC95\% 1,55-5,52), vivir en hacinamiento (HR=2,23; IC95\% 1,13-4,49) y edad (HR=0,98; IC95\% 0,95-1,00). Conclusión: Los factores de riesgo de infección hallados avalan las políticas y protocolos de protección adoptados por las autoridades sanitarias de Argentina para la población general y los programas de atención a los trabajadores de la salud en la etapa pre-vacunación.

Palabras clave: Infecciones por coronavirus. Estudios de cohortes. Factores de riesgo. Epidemiología.

Instituto Nacional de Epidemiología “Dr. Juan H. Jara" - Mar del Plata (Buenos Aires), Argentina.

Autor correspondiente: Jimena Marro. Ituzaingó, 3.520, Mar del Plata, Buenos Aires, Argentina. E-mail: jimenamarro@gmail.com. Conflicto de intereses: nada para declarar - Fuente de financiamiento: El estudio contó con financiamiento público, de la Administración Nacional de Laboratorios e Institutos de Salud Dr. Carlos Malbrán (ANLIS), Argentina. 
ABSTRACT: Objective: To establish the magnitude and risk factors for SARS-CoV-2 infection in the General Pueyrredón, Buenos Aires, Argentina: the INECOVID study. Methods: Prospective cohort designed with participants from the District general population. The follow-up period was from June $22^{\text {nd }}$ to December $18^{\text {th }}, 2020$, with a minimum appointment interval of 21 days. Data were obtained via questionnaires and serum or plasma samples. The primary event was considered as the time to seroconversion (IgG) as evidence of SARS-CoV-2 infection. The accumulated risk of infection was estimated using the Kaplan Meier method. Cox models were built with time-dependent variables. Results: 345 participants were recruited ( $\mathrm{n}=222$ women, $64.3 \%$; 123 men, 35.7\%), with a median age of 45 years in women (Interquartile range: 19) and 49 in men (Interquartile range: 26 ). 12.8\% of participants $(n=44)$ had evidence of SARS-CoV-2 infection [incidence density of 9.1 cases (women: 11.1, men: 5.1) per 10,000 person-day]. $36.4 \%$ of the cases $(n=16)$ were asymptomatic. The following factors were associated to the risk of infection: being in close contact of a confirmed COVID-19 case (HR=5.56; 95\%CI 2.85-10.83), being a health worker $(\mathrm{HR}=2.93 ; 95 \% \mathrm{CI} 1.55-5.52)$, living in crowded conditions (HR=2.23; 95\%CI 1.13-4.49), and age $(\mathrm{HR}=0.98 ; 95 \% \mathrm{CI}$ 0.95-1.00). Conclusion: The identified risk factors endorse the protection policies and protocols adopted by the Argentinian sanitary authorities for the general population and the care programs for health workers in the pre-vaccination phase.

Keywords: Coronavirus infections. Cohort studies. Risk factors. Epidemiology.

\section{INTRODUCCIÓN}

La pandemia de la enfermedad por coronavirus 2019 (COVID-19) causada por el síndrome respiratorio agudo severo (SARS-CoV-2) puso en jaque a los sistemas de salud en gran parte del mundo. Con un elevado nivel de transmisibilidad, desde su inicio a partir de la notificación del primer caso confirmado el 31 de diciembre de 2019 en Wuhan, China, la enfermedad se ha extendido de manera desproporcionada a nivel global. Argentina informó su primer caso confirmado el día 3 de marzo de $2020^{1}$.

Al 22 de junio de 2020, fecha de inicio de este estudio, se habían registrado 8.860.331 casos confirmados y 465.740 muertes a nivel global, siendo estos valores para la región de las Américas de 4.370 .519 y 221.771 respectivamente ${ }^{2}$; el total de casos confirmados en Argentina fue de 44.931, con 1.043 fallecimientos ${ }^{3}$. El gobierno argentino impulsó una estrategia de supresión temprana con el objetivo de reducir la circulación viral y evitar el crecimiento exponencial de la curva de casos, en una etapa aún pre epidémica. El día 19 de marzo, con 31 casos confirmados, se decretó el aislamiento social, preventivo y obligatorio (ASPO), de la mano de una variedad de medidas en otros sectores, como la suspensión del dictado de clases en el sistema educativo.

La emergencia de la pandemia planteó el desafío de generar investigaciones tempranas que contribuyeran a la comprensión de los patrones de transmisión de la enfermedad, su severidad, características clínicas y factores de riesgo ${ }^{4}$. En esta línea, este trabajo se basó en las recomendaciones de la Organización Mundial de la Salud (OMS) relativas 
a la elaboración de protocolos de investigación seroepidemiológica de la infección por SARS-CoV-25.

El Instituto Nacional de Epidemiología “Dr. Juan H. Jara” (INE), integrante de la Red Nacional de Laboratorios de Referencia de la Administración Nacional de Laboratorios e Institutos de Salud (ANLIS-Malbrán), se emplaza en la ciudad de Mar de la Plata, cabecera del Partido de General Pueyrredón (PGP), provincia de Buenos Aires, Argentina. Es el centro de derivación de muestras para el diagnóstico de infección por SARS-CoV-2 en la región sanitaria VIII de la provincia de Buenos Aires. En función de la pertenencia del equipo de investigadores al INE, se escogió como área de estudio el PGP. Por otra parte, al momento de la planificación de la investigación, no se había verificado transmisión comunitaria, lo cual constituía una ventaja en respecto a la posibilidad de evaluar la epidemia en una fase relativamente inicial.

El objetivo de la investigación fue establecer la magnitud y los factores de riesgo de infección por SARS-CoV-2 en el PGP, Buenos Aires, Argentina: el estudio INECOVID.

\section{MÉTODOS}

Se utilizó un diseño de cohorte prospectivo. La población estuvo constituida por voluntarios de cualquier edad y género, con domicilio real en el PGP. El PGP se ubica al sureste de la provincia de Buenos Aires, Argentina; su población total estimada para el año 2020 fue de 656.456 habitantes ${ }^{6}$.

El estudio se desarrolló en el contexto de una pandemia, por lo que su interés primordial fue realizar aportes sobre aspectos clínico-epidemiológicos que contribuyan a alcanzar un control oportuno y efectivo de la situación ${ }^{5}$. Fueron elegibles para INECOVID voluntarios de todos los rangos etarios y géneros con domicilio real en el PGP, con posibilidades de permanecer 6 meses en seguimiento y brindar su consentimiento informado firmado. Se excluyeron personas con contraindicación para la venopunción. Los participantes fueron reclutados a partir de una convocatoria abierta difundida en comunicados de prensa, anuncios en diarios y radios locales, redes sociales y correos electrónicos. Con el objetivo de incluir participantes de edad pediátrica y adolescente, se invitó a niños y adolescentes que concurrieran periódicamente al Hospital Interzonal Especializado Materno Infantil “Don Victorio Tetamanti” (HIEMI) para controles por alguna patología de base, en situaciones en que dichos controles incluyeran una extracción sanguínea.

Se propuso reclutar 300 participantes voluntarios, tomando en cuenta las capacidades locales y la disponibilidad de recursos ${ }^{5}$. El período de seguimiento fue del 22 de junio al 18 de diciembre de 2020. Desde el inicio del estudio, los participantes fueron incluidos hasta cumplir con la muestra establecida; a medida que se registraron pérdidas, se incorporaron nuevos participantes con fecha límite en el 16 de noviembre (cohorte dinámica). Los participantes concurrieron al INE en varias ocasiones durante el seguimiento, con un intervalo 
mínimo de 21 días y turnos previamente acordados por un integrante del equipo que realizó su recepción telefónica, excepto en el caso de niños y adolescentes del HIEMI.

La información se obtuvo mediante un cuestionario diseñado específicamente para este estudio, administrado en el marco de una entrevista por encuestadores previamente capacitados y muestras de sangre total obtenidas por venopunción. Las preguntas incluidas en dicho cuestionario fueron preguntas previamente validadas, tomadas de las siguientes fuentes de datos de la administración pública nacional: cuestionarios del Instituto Nacional de Estadísticas y Censos (INDEC), cuestionarios utilizados en la Encuesta Nacional de Factores de Riesgo $2018^{7}$ y Ficha de Notificación de casos sospechosos de COVID-19 ${ }^{8}$. Se tomó la propuesta de incorporación de la variable étnica al sistema de información en salud pública en tiempos de COVID-19, consensuada en el proceso de participación del diseño pre censal con el INDEC y el Tejido de Profesionales Indígenas'. Se realizó una prueba piloto del instrumento de encuesta, que consistió en la realización de entrevistas simuladas con trabajadores del INE y personas de la comunidad, con el solo fin de evaluar su adaptación. Se realizaron los ajustes necesarios en que las preguntas en que hubo dificultades de interpretación por parte del entrevistado y se confeccionó el manual del encuestador.

Los participantes cumplieron con un ayuno mínimo de dos horas para la extracción sanguínea. Las determinaciones serológicas se realizaron en el laboratorio del INE, bajo un nivel de bioseguridad II. Se utilizaron las pruebas COVIDAR IgG (autorizadas por ANMAT PM 1545-4) y COVIDAR IgM (COVIDAR IgM, ANMAT PM 1545-5), ambas codesarrolladas por CONICET, Instituto Leloir, Universidad de San Martín y Laboratorio Lemos SRL ${ }^{10}$. Estas pruebas son ensayos inmunoenzimáticos, heterogéneos, no competitivos, basados en un método indirecto para la detección in vitro de anticuerpos IgG/IgM específicos para SARS-CoV-2 en muestras de suero o plasma humanos.

La detección de ambos isotipos se efectuó en forma paralela. Frente a la detección de anticuerpos de tipo IgM, se tomó una muestra de hisopado nasofaríngeo y oro faríngeo para realización de la prueba de reacción en cadena de la polimerasa en tiempo real (reverse transcription polymerase chain reaction - RT-PCR) con el objetivo de descartar una infección activa. En tales casos, se procedió al aislamiento del participante y búsqueda activa de sus contactos estrechos, en línea con lo establecido por la estrategia de vigilancia epidemiológica nacional. En todos los casos en que se detectó IgG, se procedió a la titulación del nivel de anticuerpos, utilizando diluciones seriadas al medio, de acuerdo con las instrucciones del fabricante. El título se informó como la inversa de la última dilución que diera positiva, según la técnica.

El evento primario a observar fue el tiempo desde el ingreso a la cohorte (evento inicial) hasta la ocurrencia de la seroconversión (medida en anticuerpos IgG específicos para SARSCoV-2) considerada como evidencia serológica de infección por SARS-CoV-2 (evento final).

Las variables se organizaron en cuatro bloques: identificación del participante, datos socios demográficos, antecedentes clínico-epidemiológicos e historia de síntomas.

Se describieron las características de la línea de base; las variables continuas fueron resumidas por medio de la mediana y el rango intercuartílico, las variables categóricas a partir de las frecuencias absoluta y relativa. 
Se calculó la densidad de incidencia por sexo, utilizando el total de tiempo-persona en riesgo. Se estimó la función de riesgo acumulado por el método de Kaplan Meier estratificando por las diferentes variables consideradas fijas: edad (46 años o menos/mayor de 46 años), sexo (hombre/ mujer), nivel de educación (primario o menor/secundario/terciario o universitario), personal de salud (sí/no), hacinamiento (menos de 2 personas por cuarto/ 2 o más personas por cuarto), tratamiento inmunosupresor (sí/no), presencia de comorbilidades (sí/no). La comparación de las curvas se realizó mediante long-rank tests $(\mathrm{p}<0,05)$.

Se construyeron modelos de riesgos proporcionales de Cox (MRP) para covariables tiempo-dependientes; se estimaron los Hazard Ratio (HR) ajustados para cada una de las covariables, con un intervalo de confianza (IC) del $95 \%$. Se consideraron para la construcción del modelo aquellas variables que resultaron significativas según el log-rank test y se testearon las covariables tiempo-dependientes: situación actual (aislado/salidas indispensables/ trabajando/ exceptuado/otro) y antecedente de contacto estrecho con un caso confirmado de COVID-19.

La información registrada en los cuestionarios fue introducida por un operador de entrada de datos entrenado, en una base confeccionada en EpiInfo 7.2.4.0. Para el procesamiento de la base de datos se utilizó el lenguaje R 3.6.3 ${ }^{11}$, funciones de los paquetes tidyverse ${ }^{12}$, epiR ${ }^{13}$, survival ${ }^{14}$ y survminer ${ }^{15}$, corriendo en entorno R Studio 1.1.383.

El protocolo fue aprobado por el Comité de Ética en Investigación del Instituto Nacional de Epidemiología “Dr. Juan H. Jara”, inscripto bajo el código CE00264 en el Registro Nacional de Investigaciones en Salud (RENIS) de Argentina, acreditado por el Comité de Ética Central bajo el no 059/2019. Código: MARRO 02/2020.

\section{RESULTADOS}

Entre el 22 de junio y el 16 de noviembre de 2020, fueron reclutados 345 participantes voluntarios de la población general del PGP con edades entre 11 meses y 81 años $(n=222$ mujeres, $64,3 \%$; 123 hombres, 35,7\%). Los participantes fueron entrevistados con la periodicidad establecida; en la etapa de trabajo de campo no se registraron datos faltantes. Las pérdidas durante el seguimiento representaron el $8,0 \%(n=27)$ y se debieron al abandono del estudio, el $91,7 \%$ de los cuales lo hizo en la última visita $(n=22)$.

En la línea de base, la mediana de edad de los participantes fue de 45 años en las mujeres [Rango intercuartílico (RIQ) 19] y 49 en los hombres (RIQ 26). Con relación al género, 3 participantes $(0,8 \%)$ se adscribieron a la categoría no binaria. El 1,8\% de las mujeres $(n=4)$ y el $3,3 \%$ de los hombres $(n=4)$ se reconocieron como descendientes de pueblos originarios o afrodescendientes. El nivel de educación terciario o universitario fue el predominante en ambos sexos. El 95\% de las mujeres $(n=201)$ y el $90 \%$ de los hombres $(n=111)$ poseían una cobertura de obra social, mutual o prepaga previo al inicio del ASPO. La comorbilidad más común en ambos sexos fue la obesidad, seguida de la hipertensión arterial (Tabla 1). 
Tabla 1. Características de los participantes de INECOVID en la línea de base.

\begin{tabular}{|c|c|c|c|}
\hline \multirow[b]{2}{*}{ Variable } & \multirow[b]{2}{*}{$\mathrm{n}$} & \multicolumn{2}{|c|}{ Sexo } \\
\hline & & $\begin{array}{c}\text { Mujeres } \\
n(\%)\end{array}$ & $\begin{array}{c}\text { Hombres } \\
\text { n (\%) }\end{array}$ \\
\hline Adscripción a un grupo étnico & 342 & $4(1,8)$ & $4(3,3)$ \\
\hline \multicolumn{4}{|l|}{ Educación } \\
\hline Especial & \multirow{5}{*}{344} & $1(0,5)$ & $0(0,0)$ \\
\hline Jardín de infantes & & $0(0,0)$ & $1(0,8)$ \\
\hline Primario & & $12(5,4)$ & $5(4,1)$ \\
\hline Secundario & & $50(22,6)$ & $35(28,5)$ \\
\hline Terciario/universitario & & $158(71,5)$ & $82(66,7)$ \\
\hline \multicolumn{4}{|l|}{ Condición de actividad } \\
\hline Desocupado & \multirow{4}{*}{342} & $10(4,5)$ & $5(4,1)$ \\
\hline Jubilado & & $25(11,4)$ & $13(10,7)$ \\
\hline No trabaja & & $22(10,0)$ & $11(9,0)$ \\
\hline Ocupado & & $163(74,1)$ & $93(76,2)$ \\
\hline Cobertura de salud previo al ASPO & 345 & $210(94,6)$ & $111(90,2)$ \\
\hline Trabajador de salud & 341 & $50(22,7)$ & $10(8,3)$ \\
\hline \multicolumn{4}{|l|}{ Hacinamiento } \\
\hline Menos de 2 personas por cuarto & \multirow{3}{*}{344} & $190(86,0)$ & $106(86,2)$ \\
\hline De 2 a 3 personas por cuarto & & $29(13,1)$ & $14(11,4)$ \\
\hline Hacinamiento crítico & & $2(0,9)$ & $3(2,4)$ \\
\hline \multicolumn{4}{|l|}{ Acceso a agua potable* } \\
\hline Fuera de la vivienda, dentro del terreno & \multirow{2}{*}{345} & $6(2,7)$ & $0(0,0)$ \\
\hline Por cañería dentro de la vivienda & & $216(97,3)$ & $123(100)$ \\
\hline \multicolumn{4}{|l|}{ Situación actual } \\
\hline Trabajando, exceptuado del ASPO & \multirow{4}{*}{345} & $113(50,9)$ & $67(54,5)$ \\
\hline Salidas (compras y gestiones indispensables) & & $101(45,5)$ & $48(39,0)$ \\
\hline Aislado & & $4(1,8)$ & $3(2,4)$ \\
\hline Otra situación & & $4(1,8)$ & $5(4,1)$ \\
\hline Contacto estrecho con caso de COVID-19 & 345 & $6(2,7)$ & $5(4,1)$ \\
\hline Tabaquismo actual & 343 & $46(20,8)$ & $26(21,3)$ \\
\hline
\end{tabular}


Tabla 1. Continuación.

\begin{tabular}{l|c|c|c|}
\hline \multirow{2}{*}{ Variable } & & \multicolumn{2}{|c}{ Sexo } \\
\cline { 3 - 4 } & & $\begin{array}{c}\text { Mujeres } \\
\mathrm{n}(\%)\end{array}$ & $\begin{array}{c}\text { Hombres } \\
\mathrm{n}(\%)\end{array}$ \\
\hline Comorbilidades & & & $25(20,3)$ \\
\hline Hipertensión arterial & 345 & $26(11,7)$ & $7(5,7)$ \\
\hline Asma/EPOC & 345 & $15(6,8)$ & $7(5,7)$ \\
\hline Otras enfermedades cardiovasculares & 345 & $9(4,1)$ & $3(2,4)$ \\
\hline Diabetes mellitus & 345 & $8(3,6)$ & $3(2,4)$ \\
\hline Enfermedad oncológica & 345 & $5(2,3)$ & $5(4,1)$ \\
\hline Inmunodeficiencia & 344 & $4(1,8)$ & $0(0,0)$ \\
\hline Enfermedad renal crónica & 345 & $1(0,5)$ & $1(0,8)$ \\
\hline Tuberculosis & 345 & $1(0,5)$ & $29(24,0)$ \\
\hline Obesidad & 342 & $42(19,0)$ & \\
\hline
\end{tabular}

ASPO: aislamiento social, preventivo y obligatorio. *El $91 \%$ de los participantes de ambos sexos obtiene el agua de la red pública (202 mujeres, 112 hombres); el resto lo hace por medio de perforación con bomba a motor

El 12,8\% de los participantes ( $n=44)$ tuvo un resultado reactivo de anticuerpos de tipo IgG durante el seguimiento, lo que arrojó una densidad de incidencia de 9,1 casos por 10.000 personas-día (mujeres: 11,1 , hombres: 5,1 casos por 10.000 personas-día).

El 50\% de los casos $(n=22)$ se detectaron entre las semanas epidemiológicas (SE) 35 y 46, luego de haber entrado la ciudad en la fase 3, en función de la situación sanitaria caracterizada por un aumento de la circulación viral comunitaria y antecedentes de ocurrencia de brotes de COVID-19 en instituciones de salud y establecimientos de estancia prolongada (Figura 1).

El 36,4\% de los participantes que mostraron evidencia de infección $(n=16)$ no presentaron síntomas en el período inmediatamente anterior. Entre quienes tuvieron al menos un síntoma $(63,6 \%, \mathrm{n}=28)$, los más frecuentes fueron cefaleas $(40,9 \%, \mathrm{n}=18)$; mialgias $(36,4 \%$, $\mathrm{n}=16)$; odinofagia y anosmia $(31,8 \%, \mathrm{n}=14)$; tos y fatiga $(29,5 \%, \mathrm{n}=13)$. Únicamente el $22,7 \%$ de los que tuvieron síntomas $(\mathrm{n}=10)$ informaron de fiebre.

Sólo el $31,8 \%$ de los participantes con IgG reactiva $(n=14)$ se había realizado un hisopado; 10 de ellos $(71,4 \%)$ obtuvieron un resultado de RT-PCR detectable.

En $17(38,6 \%)$ de los 44 participantes que presentaron IgG positiva, fue detectada IgM en forma sincrónica a la detección de IgG. Sólo en un caso, la detección de IgM ocurrió en la visita previa a la detección de IgG. Respecto de los títulos de anticuerpos hallados, los mismos oscilaron entre 50 y 12.800 , con una mediana de 400 . Al respecto, se observó que en las personas de 46 años o menos, solo 7 (23,3\%) exhibieron títulos superiores a la mediana, en tanto que, en los mayores de 46 años, ese porcentaje fue del $57,1 \%$. 


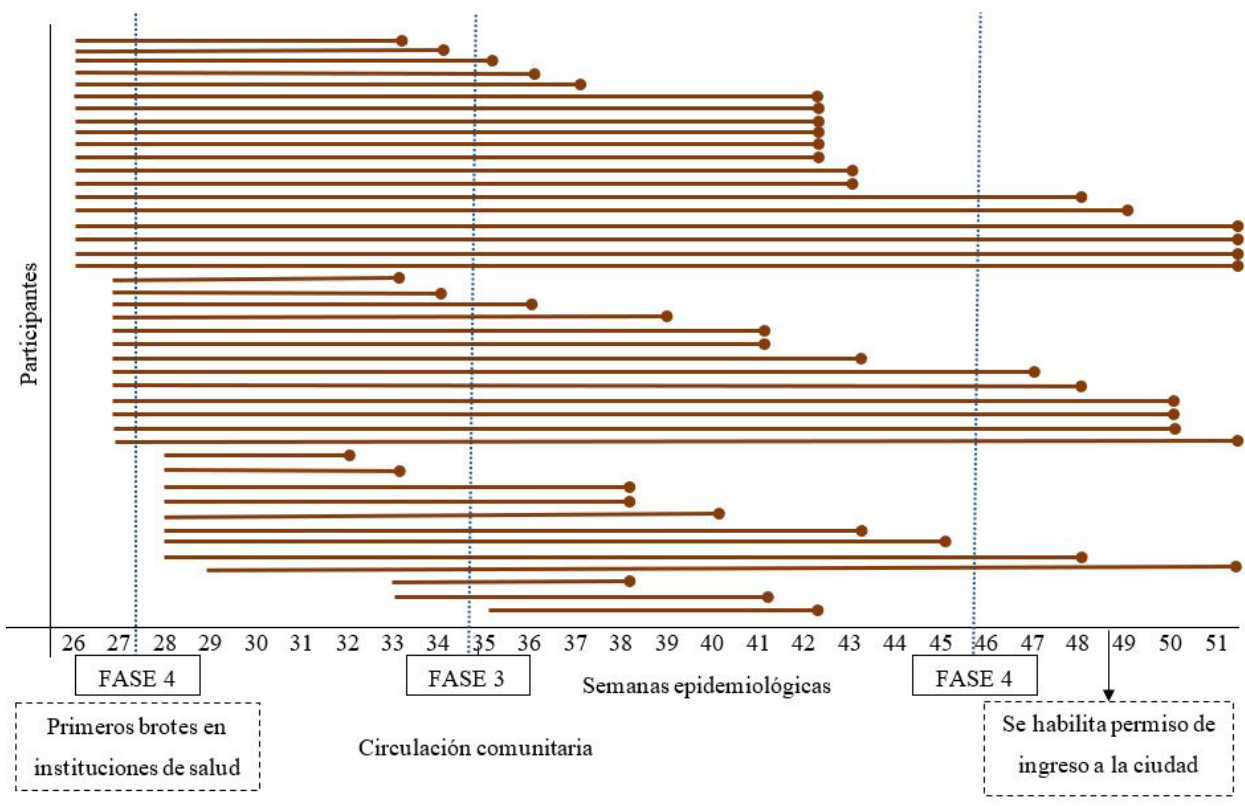

Figura 1. Dinámica de infección por SARS-CoV-2 en participantes de INECOVID en la línea de tiempo, junio-diciembre de 2020 ( $n=44)$.

Ninguno de los participantes requirió internación ni falleció durante el seguimiento.

El riesgo acumulado de infección por SARS-CoV-2 se muestra en la Figura 2. Los factores estadísticamente asociados con la probabilidad de seroconversión (log-rank test $<0,05)$ fueron sexo, edad, hacinamiento y personal de salud: las mujeres, los participantes con 46 años o menos, el personal de salud y quienes viven con dos o más habitantes por cuarto tuvieron una probabilidad mayor de seroconversión.

En el análisis múltiple se observó que tener antecedente de contacto estrecho con un caso confirmado de COVID-19 incrementó más de 5 veces el riesgo de infección $[\mathrm{HR}=5,6$ (IC95\% 2,9-10,8)]. Este riesgo fue aproximadamente el doble para quienes viven en situación de hacinamiento $[\mathrm{HR}=2,3$ (IC95\% 1,1-4,5)]. Aquellas personas que trabajan en salud presentaron aproximadamente tres veces el riesgo de aquellas que no $[\mathrm{HR}=2,9$ (IC95\% 1,6$5,5)]$. Por otra parte, por cada año de incremento de la edad, hubo una disminución aproximada del 2\% en el riesgo de infección [HR=0,98 (IC95\% 0,95-1,0)] (Figura 3). El modelo ajustado presentó una concordancia muy buena $(0,773)$, en tanto que el análisis de residuos mostró que se cumplieron los presupuestos del modelo.

\section{DISCUSIÓN}

En este estudio de cohortes en momentos tempranos de pandemia, la densidad de incidencia fue de 9,1 casos de infección por SARS-COV-2 por 10.000 personas-día. 


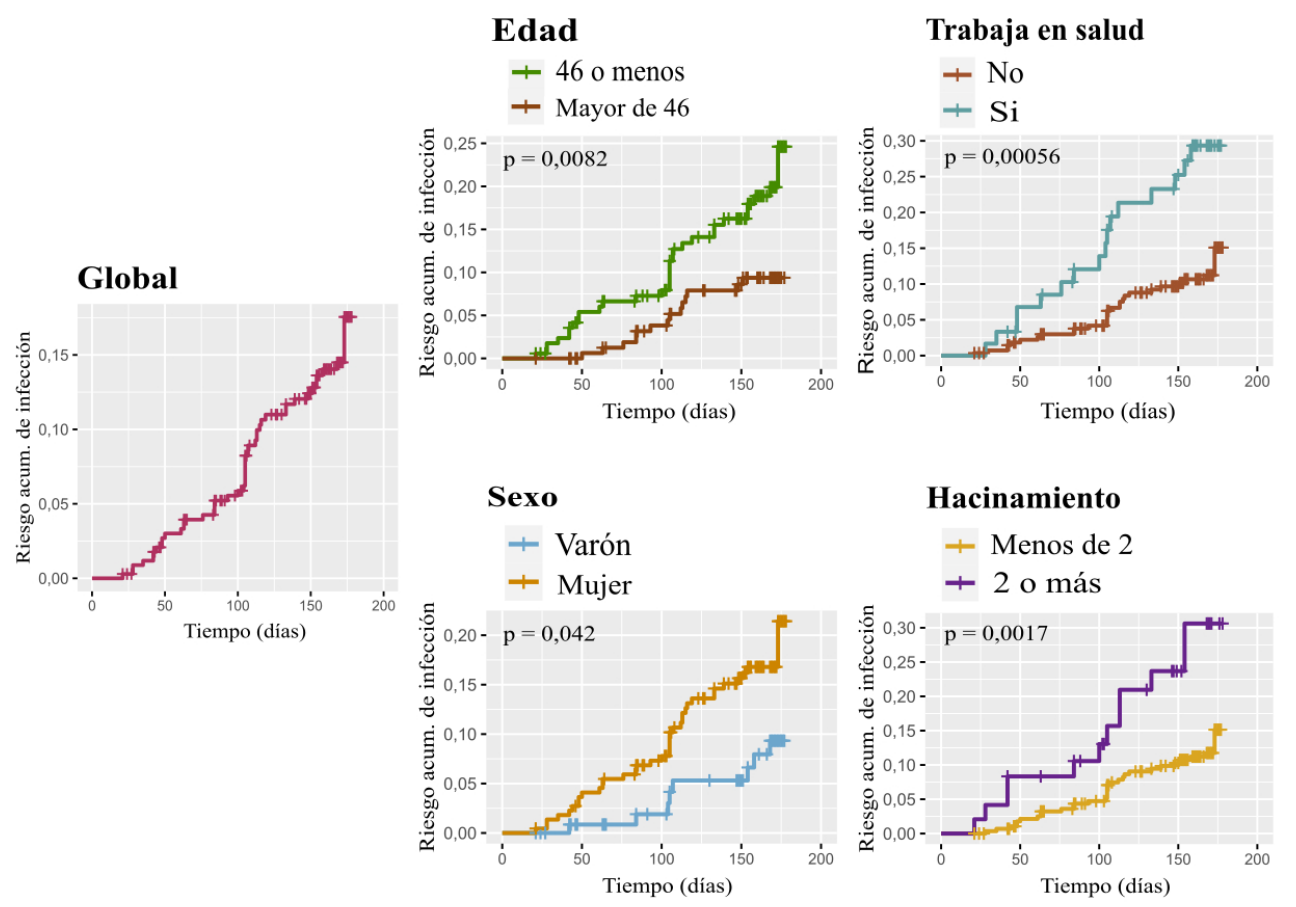

Figura 2. Riesgo acumulado de infección por SARS-CoV-2 global y según sexo, edad, hacinamiento y actividad laboral en participantes de INECOVID, junio-diciembre de 2020.

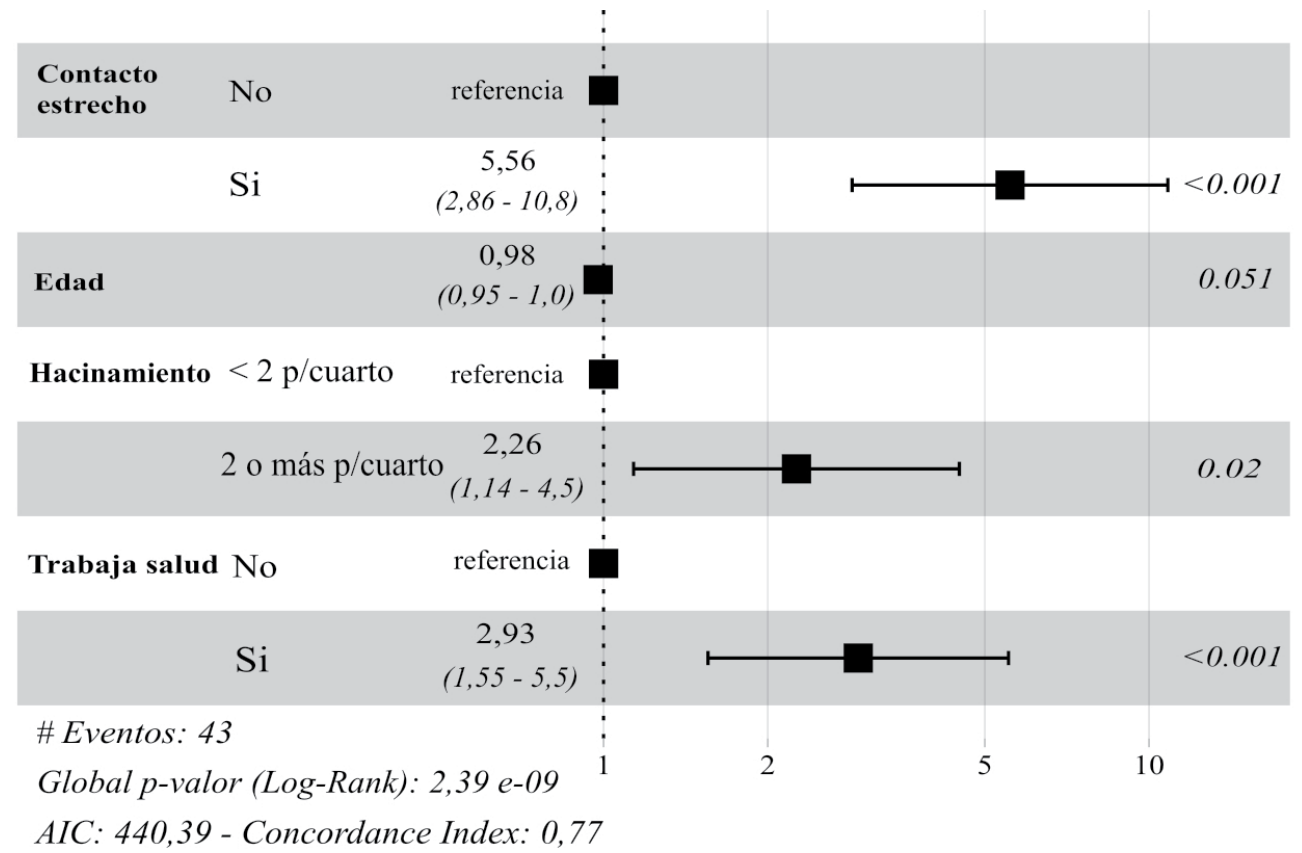

Figura 3. Hazard Ratios ajustados de los factores de riesgo de infección por SARS-CoV-2 en participantes de INECOVID, junio-diciembre de 2020. 
Por otra parte, ser contacto estrecho de un caso confirmado, ser trabajador de salud, vivir en situación de hacinamiento y edad resultaron los principales factores asociados a la infección.

La proporción de personas con evidencia de infección y curso asintomático hallada en nuestro trabajo $(36,4 \%)$ fue similar a la encontrada en otras investigaciones: experiencias como la de $\mathrm{Vo}^{\prime}$ en Italia, conducidas en la población general durante el bloque de 14 días impuesto por las autoridades, encontraron $42,5 \%$ de las infecciones confirmadas por SARS-CoV-2 como asintomáticas ${ }^{16}$; otro estudio llevado a cabo en Islandia reportó un $43 \%{ }^{17}$. En una investigación realizada en el crucero Diamond Princess, al momento de realizar las pruebas, el $46,5 \%$ de los que obtuvieron resultados positivos estaban asintomáticos ${ }^{18}$. Se resalta entonces una de las principales utilidades de los ensayos serológicos: el testeo longitudinal de una población determinada permite estimar la incidencia de exposición de la población al virus, incluso de aquellos individuos que hubieran cursado la infección en forma asintomática.

Respecto de los factores de riesgo de infección por SARS-CoV-2 en esta cohorte, el antecedente de contacto estrecho de un caso confirmado de COVID-19, ser trabajador de salud y vivir en situación de hacinamiento fueron los más relevantes: el primero fue el de mayor fuerza de asociación, sextuplicando el riesgo. Resultados similares se obtuvieron en un análisis de regresión logística condicional múltiple sobre los tipos de contacto: el contacto en el hogar (OR 6,3) y viajar juntos en automóvil (OR 7,1) se asociaron significativamente con la infección ${ }^{19}$. Ya en las primeras etapas de la epidemia en China, el antecedente de contacto estrecho con un caso de COVID-19 fue señalado como un factor de riesgo de enfermedad ${ }^{20-22}$, por lo tanto, incluido entre las medidas de prevención de brotes ${ }^{23}$.

Con relación a la condición de trabajador de salud, desde etapas tempranas de la pandemia fue subrayada a nivel internacional la preocupación por el riesgo aumentado de infección por SARS-CoV-2 en este subgrupo de la población, al constituir la primera línea de batalla en la lucha contra la epidemia. Lo observado en este estudio concuerda con lo evidenciado por investigaciones llevadas a cabo en diferentes países ${ }^{24-26}$.

El hacinamiento representa el cociente entre la cantidad de personas y el número total de habitaciones para dormir en el hogar. En nuestra cohorte, cuando este cociente era de 2 o más personas por cuarto, el riesgo de infección se duplicó. Esto resulta lógico, dado que en esas circunstancias se reducen las posibilidades de aislamiento ante un caso positivo entre los convivientes, atentando contra la necesidad de reducir la concentración de partículas portadoras de virus en el aire y, en consecuencia, el número de personas expuestas. Estos hallazgos son consistentes con otros estudios que encontraron que el hacinamiento es uno de los factores socioeconómicos que se asocian a un mayor riesgo de enfermedad por COVID ${ }^{27-29}$. Al tratarse de una enfermedad respiratoria transmitida por aerosoles, este vínculo ya se había evidenciado para enfermedades como la tuberculosis y la influenza ${ }^{30,31}$.

Con relación a las diferencias por sexo, en lo que respecta a la respuesta de anticuerpos, existen trabajos que mostraron mayores títulos en mujeres que en hombres después 
de una infección grave por COVID-1932. Aunque en nuestro trabajo encontramos una mayor probabilidad de seroconversión en las mujeres, no se observó dicho riesgo en el análisis ajustado.

Aunque la mayoría de las investigaciones que abordan la relación entre la presencia de comorbilidades y COVID-19 hayan destacado el impacto en términos de progresión de la enfermedad y desenlaces fatales ${ }^{33,34}$, también han sido subrayados los mecanismos fisiopatológicos involucrados en el aumento del riesgo de infección por SARS-CoV-2 en pacientes con comorbilidades ${ }^{35}$. En nuestro estudio, no encontramos diferencias significativas en el riesgo de enfermar en participantes con comorbilidades, incluyendo enfermedad cardíaca, diabetes mellitus, asma/EPOC, inmunodeficiencia, enfermedad oncológica, obesidad y enfermedad renal crónica. Las medidas de protección sanitaria implementadas en Argentina durante el primer año pandémico, coincidente con el período del estudio, incluyeron entre sus prioridades el resguardo de los grupos vulnerables, como las personas de edad avanzada y con comorbilidades. Nuestra hipótesis es que esto puede haber influido en los resultados observados en esta investigación, al tratarse de grupos que han tenido menor movilidad y, por lo tanto, una exposición reducida al virus.

La principal fortaleza de nuestro estudio radica en el extenso seguimiento realizado con los participantes y en la frecuencia de medición, pautadas cada 21 días. El primero permitió monitorear la probabilidad de seroconversión en distintos escenarios pandémicos; la segunda, ajustar cada evento a una ventana temporal acotada. A su vez, la tasa de abandonos fue baja. En ese sentido, se trabajó intensamente para evitar pérdidas de seguimiento, por medio de repetidos llamados telefónicos y comunicaciones vía WhatsApp en caso de ausencia al turno programado.

Como limitaciones, los participantes no fueron seleccionados mediante muestreo probabilístico, por lo que los resultados no deben extrapolarse a la población general. Con relación a la población general del PGP ${ }^{36}$, en nuestra muestra estuvieron sobrerrepresentados el grupo de edad entre 35 y 64 años, las mujeres, las personas con nivel de educación terciaria o universitaria y aquellos con algún tipo de cobertura de salud (obra social, mutual o prepagas); mientras que las personas desocupadas se encontraron sub representadas. En función del número final de participantes, considerando como covariable de interés el antecedente de contacto estrecho, por ser tiempo dependiente y en función de los HR hallados, los cálculos de potencia oscilaron entre 74,10 y $74,26^{37}$.

Respecto de las determinaciones serológicas en sí, la técnica empleada resultó robusta, tanto para la detección de casos que fueron positivos por RT-PCR, como para aquellos que se auto percibieron como asintomáticos. Asimismo, un informe publicado por los desarrolladores del ensayo sostiene que las titulaciones de IgG totales contra spike empleando COVIDAR son altamente informativas para estimar la capacidad neutralizante de dichos anticuerpos ${ }^{38}$.

En nuestra cohorte, las personas mayores de 46 años exhibieron mayor proporción de títulos altos de anticuerpos. Esto es coincidente con lo encontrado en otro estudio llevado a cabo en nuestro país, con la misma técnica, donde las mediciones de anticuerpos también se correlacionaron con la edad, mostrando los niveles más altos de 
anticuerpos asociados a pacientes mayores. En este mismo estudio, se observó seroconversión sincrónica de IgG e IgM en la mayoría de los casos (72\%), seroconversión de IgM previa a IgG en el $21 \%$ de los pacientes, mientras que la IgG apareció antes que IgM en el 7\% de los pacientes. Estos resultados también van en la misma dirección de los obtenidos en nuestra cohorte, con la salvedad que, en nuestro caso, no pudimos reconstruir esta última situación, dado que al momento de ser IgG positiva, nuestros participantes salían de la cohorte ${ }^{39}$.

Como conclusión, la respuesta de anticuerpos captó un tercio de infectados asintomáticos no detectados en el sistema de vigilancia epidemiológica, lo que implica que la infección por SARS-CoV-2 pudo ser mayor al número de casos confirmados oficiales. Los factores de riesgo de infección hallados en esta cohorte avalan las políticas y protocolos de protección adoptados por las autoridades sanitarias de Argentina para la población general, así como los programas de cuidado de los trabajadores de salud en la etapa pre-vacunación.

\section{AGRADECIMIENTOS}

Agradecemos a los colaboradores de INECOVID: Gabriel Amezqueta, Sebastian Bienaimé, Laura Caldera, Ramiro Dana Smith, Héctor Garcialoredo, Francisco González Espinosa, Federico Grosso, Mercedes Hoffmann, Silvina Lavayén, Valeria Macías Lainez, Jimena Menéndez, Indira Monte, Lisa Roques, Rodrigo Sabuda, Martina Snitman, Mariel Varela, Marcelo Zotta, Alicia Lawrynowicz, Irene Pagano y Osvaldo Uez (INE). A Diego Rodríguez y Hugo Casarsa (HIEMI) y a Andrea Gamarnik (IIBBA-CONICET) por la donación de COVIDAR.

\section{REFERENCIAS}

1. Ministerio de Salud de la Nación Argentina. Nuevo Coronavirus (COVID-19). Reporte diario [Internet]. Buenos Aires: MSAL; 2020 [accedido el 5 mar. 2020]. Disponible el: https://www.argentina.gob.ar/ coronavirus/informe-diario

2. World Health Organization. Coronavirus disease 2019 (COVID-19). Situation report - 154 [Internet]. 2020 [accedido el 12 abr. 2021]. Disponible el: https: / / www.who.int/docs/default-source/coronaviruse/ situation-reports / 20200622-covid-19-sitrep-154. pdf?sfvrsn=d0249d8d_2
3. Argentina. Ministerio de Salud. Nuevo Coronavirus (COVID-19). Reporte diario matutino Nro 199. Situación de COVID-19 en Argentina [Internet]. 2020 [accedido el 22 jun. 2020]. Disponible el: https: / / www.argentina.gob.ar/sites/default/files / 22-06-20_ reporte-matutino-covid-19.pdf

4. World Health Organization. Coronavirus disease (COVID-19) technical guidance: the unit studies: early investigations protocols [Internet]. 2020 [accedido el 3 abr. 2020]. Disponible el: https:/ / www.who. int/emergencies / diseases/novel-coronavirus-2019/ technical-guidance/ early-investigations 
5. Organización Mundial de la Salud. Protocolo para estudios seroepidemiológicos poblacionales sobre la COVID-19, con estratificación por edades. Versión 1.1 [Internet]. 2020 [accedido el 12 jun. 2020]. Disponible el: https://apps.who. int/iris/bitstream/handle/10665/331540/

WHO-2019-nCoV-Seroepidemiology-2020.1-spa. pdf? sequence $=1$ \&isAllowed $=\mathrm{y}$

6. Instituto Nacional de Estadísticas y Censos. Proyecciones por departamento [Internet]. 2021 [accedido el 30 jun. 2021]. Disponible el: https: / / www.indec.gob.ar/indec/ web/Nivel4-Tema-2-24-119

7. Instituto Nacional de Estadística y Censos. $4^{\circ}$ Encuesta Nacional de Factores de Riesgo. Resultados definitivos. $1^{\text {a }}$ ed. Burnos Aires: Secretaria de Gobierno de Salud de la Nación; 2019 [accedido el 3 jun. 2021]. Disponible el: https:/ / www.indec.gob.ar/ftp/cuadros/publicaciones/ enfr_2018_resultados_definitivos.pdf

8. Argentina. Ministerio de Salud. Ficha de notificación, investigación epidemiológica y solicitud de estudios de laboratorio de caso sospechosos de nuevo coronavirus COVID-19. Buenos Aires; 2020.

9. Azpiroz Cleñan V. Tejido de Profesionales Indígenas en Argentina. Salud intercultural. Incorporación de la variable étnica en el sistema de información de salud pública en tiempos de COVID-19; 2020

10. COVID AR IgG. Enzimoinmunoensayo (ELISA) para la detección de anticuerpos IgG específicos contra el virus SARS-CoV-2 en suero o plasma humano. Buenos Aires: Laboratorio LEMOS S.R.L. [accedido el 9 nov. 2021]. Disponible el: https://portal-coronavirus. gba.gob.ar/sites/default/files/Inserto\%20KIT\%20 COVID\%20AR\%20IgG.pdf

11. The Core Project for Statistical Computing. Team. [accedido el 29 oct. 2021]. Disponible el: http:// www.R-project.org

12. Wickham H, Averick M, Bryan J, Chang W, McGowan LDA, François R, et al. Welcome to the tidyverse. Journal of Open Source Software 2019; 4: 1686. https: / / doi.org/10.21105/joss.01686

13. Stevenson M, Sergeant E, Nunes T, Heuer C, Marshall J, Sanchez J, et al. Pachage 'epiR': Tools for the analysis of epidemiological data; 2021. [accedido el 29 out. 2021]. Disponible el: https:/ / cran.r-project.org/web/ packages/epiR/epiR.pdf

14. Therneau TM. A package for survival analysis in $R$. $\mathrm{R}$ package version 3.2-13; 2021. [accedido el 29 out. 2021]. Disponible el: https:/ CRAN.R-project.org/ package $=$ survival

15. Kassambara A, Kosinski M, Biecek P, Fabian S. Package 'survminer': Drawing survival curves using 'ggplot2'; 2021. [accedido el 29 out. 2021]. Disponible el: https: / cran.r-project.org/web/packages/survminer/ survminer.pdf
16. Lavezzo E, Franchin E, Ciavarella C, CuomoDannenburg G, Barzon L, Del Vecchio C, et al. Suppression of a SARS-CoV-2 outbreak in the Italian municipality of Vo'. Nature 2020; 584(7821): 425-9. https: / / doi.org/10.1038/s41586-020-2488-1

17. Gudbjartsson DF, Helgason A, Jonsson H, Magnusson OT, Melsted P, Norddahl GL, et al. Spread of SARS-CoV-2 in the Icelandic population. N Engl J Med 2020; 382(24): 2302-15. https:/ / doi.org/10.1056/NEJMoa2006100

18. Moriarty LF, Plucinski MM, Marston BJ, Kurbatova EV, Knust B, Murray EL, et al. Public health responses to COVID-19 outbreaks on cruise ships - Worldwide, February-March 2020. MMWR Morb Mortal Wkly Rep 2020; 69(12): 347-52. http:// doi.org/10.15585/ mmwr.mm6912e3

19. Bi Q, Wu Y, Mei S, Ye C, Zou X, Zhang Z, et al. Epidemiology and transmission of COVID-19 in 391 cases and 1286 of their close contacts in Shenzhen, China: a retrospective cohort study. Lancet Infect Dis 2020; 20(8): 911-9. https:// doi.org/10.1016/S1473-3099(20)30287-5. Erratum in: Lancet Infect Dis 2020; 20(7): e148. http: / / doi.org/10.1016/S1473-3099(20)30369-8

20. Expert Group on Prevention and Control of Novel Coronavirus Pneumonia of the Chinese Preventive Medicine Association. The latest understanding of the epidemiological characteristics of new coronavirus pneumonia. Chinese Journal of Epidemiology 2020; 41(2): 139-44. https: / doi. org/10.3760/cma.j.issn.0254-6450.2020.02.002

21. Chan JFW, Yuan S, Kok KH, To KKW, Chu H, YangJ, et al. A familial cluster of pneumonia associated with the 2019 novel coronavirus indicating person-to-person transmission: a study of a family cluster. Lancet 2020; 395(10223): 51423. https: / / doi.org/10.1016/S0140-6736(20)30154-9

22. Li Q, Guan X, Wu P, Wang X, Zhou L, Tong Y, et al. Early transmission dynamics in Wuhan, China, of novel coronavirus-infected pneumonia. N Engl J Med 2020; 382(13): 1199-207. https://doi. org/10.1056/ NEJMoa2001316

23. Chu DK, AklEA, Duda S, Solo K, Yaacoub S, Schünemann HJ, et al. Physical distancing, face masks, and eye protection to prevent person-to-person transmission of SARS-CoV-2 and COVID-19: a systematic review and meta-analysis. Lancet 2020; 395(10242): 1973-87. https: / doi.org/10.1016/ S0140-6736(20)31142-9

24. Mhango M, Dzobo M, Chitungo I, Dzinamarira T. COVID-19 risk factors among health workers: a rapid review. Safety and Health at Work 2020; 11(3): 262-5. https: / / doi.org/10.1016/j.shaw.2020.06.001

25. Nguyen LH, Drew DA, Graham MS, Joshi AD, Guo CG, Ma W, et al. Risk of COVID-19 among front-line healthcare workers and the general community: a prospective cohort study. The Lancet Public Health 2020; 5(9): e47583. https: / / doi.org/10.1016/S2468-2667(20)30164-X 
26. Milani GP, Bianchetti MG, Togni G, Schoenenberger AW, Muggli F. SARS-CoV-2 Ig G among Healthcare Workers and the General Population. Pathogens 2021; 10(4): 465. https:// doi.org/10.3390/pathogens10040465

27. Sturrock BRH, Chevassut EI, Shahvisi A, Chevassut TJT. Impact of affluence on the local spread of SARSCoV2 during the first wave of the COVID-19 pandemic. Public Health Pract (Oxf) 2021; 2: 100141. https:/ / doi.org/10.1016/j.puhip.2021.100141

28. Ahmad K, Erqou S, Shah N, Nazir U, Morrison AR, Choudhary G, et al. Association of poor housing conditions with COVID-19 incidence and mortality across US counties. PLoS One 2020; 15(11): e0241327. https: / / doi.org/10.1371/journal.pone.0241327

29. Harlem G. Descriptive analysis of social determinant factors in urban communities affected by COVID-19. J Public Health (Oxf) 2020; 42(3): 466-9. https: / / doi. org/10.1093/pubmed/fdaa078

30. Krieger J, Higgins DL. Housing and health: time again for public health action. Am J Public Health 2002; 92(5): 758-68. https: / / doi.org/10.2105/ajph.92.5.758

31. Enarson DA, Beyers N, Zhang LX. The tuberculosis pandemic today: routes of transmission and new target groups. Scand J Infect Dis 2001; 33(1): 9-12. https: / / doi.org/10.1080/003655401750064013

32. Zeng F, Dai C, Cai P, Wang J, Xu L, Li J, et al. A comparison study of SARS-CoV-2 IgG antibody between male and female COVID-19 patients: a possible reason underlying different outcome between sex. J Med Virol 2020; 92(10): 2050-4. https:// doi. org/10.1002/jmv.25989

33. Cheng S, Zhao Y, Wang F, Chen Y, Kaminga AC, Xu H. Comorbidities' potential impacts on severe and nonsevere patients with COVID-19: a systematic review and meta-analysis. Medicine 2021; 100(12): e24971. https: / / doi.org/10.1097/md.0000000000024971

34. Hoang T, Tran TAT. Comparison of comorbidities in relation to critical conditions among coronavirus disease 2019 patients: a network meta-analysis. Infect Chemother 2021; 53(1): 13. https:/ / doi.org/10.3947/ic.2020.0136

35. Alyammahi SK, Abdin SM, Alhamad DW, Elgendy SM, Altell AT, Omar HA. The dynamic association between COVID-19 and chronic disorders: an updated insight into prevalence, mechanisms and therapeutic modalities. Infect Genet Evol 2021; 87: 104647. https: / / doi.org/ 10.1016/j.meegid.2020.104647

36. Instituto Nacional de Estadísticas y Censos. Censo Nacional de Población y viviendas [Internet]2010. Base de datos REDATAM [accedido el 1 jul. 2021]. Disponible el: https://redatam.indec.gob.ar/argbin/RpWebEngine. exe $/$ PortalAction? \&MODE $=$ MAIN\&BASE $=$ CPV2010B
\&MAIN=WebServerMain.inl\&_ga $=2.37484064 .18736$ 64593.1548683890-346371981.1548683890

37. Qiu W, Chavarro J, Lazarus R, Rosner B, Ma J. powerSurvEpi: power and sample size calculation for survival analysis of epidemiological studies. R package version 0.1 .3 ; 2021. [accedido el 1 nov. 2021]. Disponible el:https:/ /CRAN.R-project.org/package=powerSurvEpi

38. Ojeda DS, Ledesma MMGL, Pallarés HM, Navarro GSC, Sánchez L, Villordo SM, et al. COVIDAR. Detección y titulación de anticuerpos anti-spyke y neutralizantes para la infección con SARS-CoV-2 [Internet] 2021 [accedido el 14 jul. 2021]. Disponible el: https:/ / www.argentina.gob.ar/sites/default/files/ informe_covidar_titulaciones_igg_2021.01.20.pdf

39. Ojeda DS, Ledesma MMGL, Pallarés HM, Navarro GSC, Sanchez L, Perazzi B, et al. Emergency response for evaluatingSARS-CoV-2 immune status, seroprevalence and convalescent plasma in Argentina. PLoS Pathog 2021; 17(1): e1009161. https://doi.org/10.1371/journal.ppat.1009161

Recibido el: 29/07/2021

Revisado el: 20/08/2021

Aceptado el: 21/09/2021

Preprint el: 23/09/2021

https://preprints.scielo.org/index.php/scielo/

preprint/view/2984

Contribuciones de los autores: Marro, M.: Administración del proyecto, Análisis formal, Conceptuación, Escrita-primera redacción, Escrita - revisión y edición, Investigación, Metodología, Recursos, Supervisión. Ballejo, C.: Análisis formal, Conceptuación, Consultoría de datos, Escrita revisión y edición, Investigación, Metodología, Software, Supervisión, Validación, Visualización. Aguirre, M.F.: Análisis formal, Conceptuación, Escrita - primera redacción, Escritura - revisión y edición, Investigación, Metodología, Supervisión, Validación, Visualización. de San Martín, M.E.: Análisis formal, Consultoría de datos, Escrita primera redacción, Escrita - revisión y edición, Investigación, Metodología. López Miranda, L.: Análisis formal, Conceptuación, Escrita - revisión y edición, Investigación, Metodología, Obtención de financiamiento, Recursos. Poncet, V.: Análisis formal, Escrita - revisión y edición, Investigación, Metodología, Recursos. Silva, A.: Administración del proyecto, Análisis formal, Conceptuación, Escritura - primera redacción, Escrita - revisión y edición, Investigación, Metodología, Obtención de financiamiento, Recursos, Supervisión, Visualización. 